\title{
In Situ Characterization of Interfacial and Bulk Properties of Lithium Polymer Batteries Using 4-Probe DC Techniques
}

\author{
Meilin Liu, ${ }^{*}$ Steven J. Visco, ${ }^{\dagger}$ and Lutgard C. De Jonghe ${ }^{\dagger}$ \\ * School of Materials Science and Engineering \\ Georgia Institute of Technology \\ Atlanta, Georgia 30332-0245 \\ ${ }^{\dagger}$ Materials Sciences Division \\ Lawrence Berkeley Laboratory \\ University of California \\ Berkeley, CA 94720 USA
}

November 1992

This work was supported by the Assistant Secretary for Conservation and Renewable Energy, Deputy Assistant Secretary for Utility Technologies, Office of Energy Management, Advanced Utility Concepts Division of the U.S. Department of Energy under Contract No. DE-AC03-76SF00098. 


\title{
IN SITU CHARACTERIZATION OF INTERFACIAL AND BULK PROPERTIES OF LITHIUM POLYMER BATTERIES USING 4-PROBE DC TECHNIQUES
}

\author{
MEILIN LIU*, STEVEN J. VISCO**, AND LUTGARD C. DE JONGHE** \\ *School of Materials Science and Engineering, Georgia Institute of Technology, Atlanta, \\ Georgia 30332-0245. \\ ** University of California, Lawrence Berkeley Laboratory, Materials Science Division, \\ Berkeley, CA 94720.
}

\begin{abstract}
The use of transient and steady-state 4-probe techniques for the evaluation of the limitations to performance in solid-state electrochemical devices is described. The application of sequential, bipolar square-wave current pulses to a solid polymer cell with two internal reference electrodes allows separation of the polarization behavior of the various components of the cell, and indicates the rate limiting processes in the cell. Careful design of the experimental cell and appropriate selection of current pulse width allows evaluation of fundamental kinetic and transport properties, as well as activation parameters. Steady state constant-current measurements using 4-probe cells allows in situ observation of interface stability and/or polarization as a function of time as the cell is cycling.
\end{abstract}

\section{INTRODUCTION}

The optimization of solid-state devices such as solid polymer batteries, sensors, and solid oxide fuel cells often involves careful investigations of bulk and interfacial properties of the devices. In thin-film devices where electrolyte thickness is minimized, interfacial phenomena often are the controlling factors in device performance. Although interfacial phenomena can be studied by techniques such as impedance spectroscopy, in situ investigations are often more effective in identifying limitations to performance. In this paper a technique is described for investigation of solid-state electrochemical devices using 4 potential probes and a sequential pulse technique. A thin-layer 4 probe cell has been designed that allows for precise positioning of two internal reference electrodes between the negative and positive electrodes in a functioning solid-state cell. The thin-layer cell allows in situ determination of Li/PEO interfacial resistance, electrolyte resistivity, and positive electrode polarization during charge and discharge cycling as a function of current density and temperature.

\section{EXPERIMENTAL}

A schematic view of a solid-state 4-probe cell and its equivalent circuit is shown in figure 1. The actual assembly of the thin-film cell is accomplished as shown in figure 2. Commercial lithium foils of approximately 100 microns are used as the negative electrode, while experimental Li foils of approximately $30 \mu \mathrm{m}$ (Cyprus Foote Mineral Co.) are used for the reference electrodes. Thin insulating sheets of polyester terephthalate (PET) (Hruechst Celanese) approximately $20 \mu \mathrm{m}$ thick are used to prevent shorting of the reference electrodes. 
Double-sided adhesive films $(30 \mu \mathrm{m})$ are used to position each successive layer as the cell is constructed so that the relative positions of the electrode do not shift after assembly. Electrical connections to the electrodes are made by thin copper tape backed with electrically conductive adhesive (3M). Once constructed, the 4-probe cells are exceedingly durable and withstand repeated cycling at various temperatures without shorting of the reference electrodes.

Polyethylene oxide (PEO) and amorphous PEO (aPEO) electrolytes and composite cathodes were prepared as described previously1,2,3. Polyorganodisulfide electrodes have been described earlier ${ }^{1,2,3}$, and the nomenclature of $X 1$ and $X 5$ is used here for polydimercaptothiadiazole and polytrithiocyanuric acid, respectively. Galvanostatic control of the cell including transient techniques is accomplished through computer control of a PAR 173 (or 371) Potentiostat/Galvanostat using a Data Translation 2801-A board and software written in ASYST language. The program written in ASYST was used to generate the bipolar current pulse as shown in figure 3 and to simultaneously acquire the potential response of the cell at the four probes at $t=\tau_{0}$. Furthermore, the program monitors the potential response at the four probes under conditions of steady-state, constant current control as used in conventional battery cycle testing.
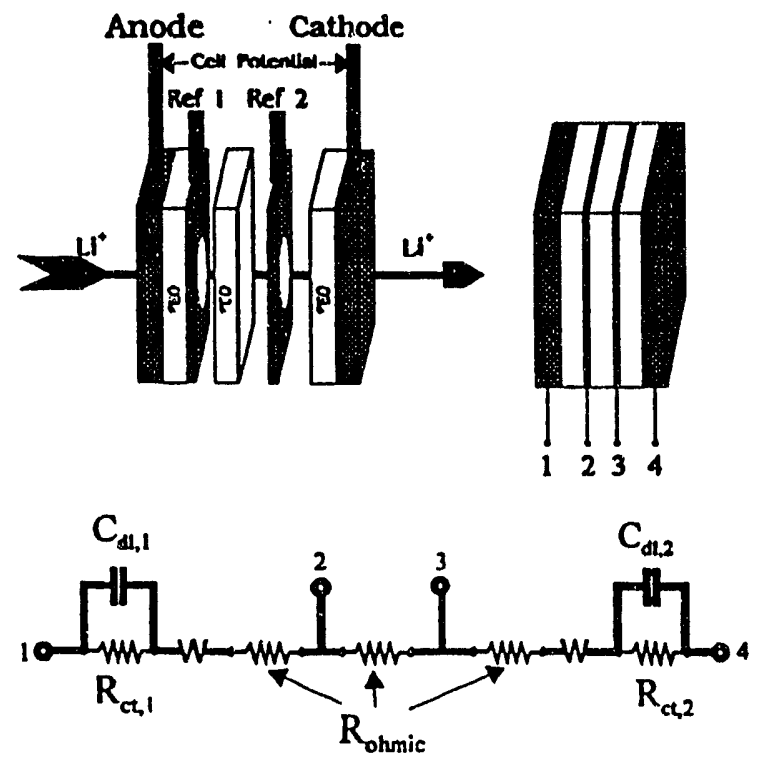

Figure 1. Solid-state four probe cell and corresponding equivalent circuit.

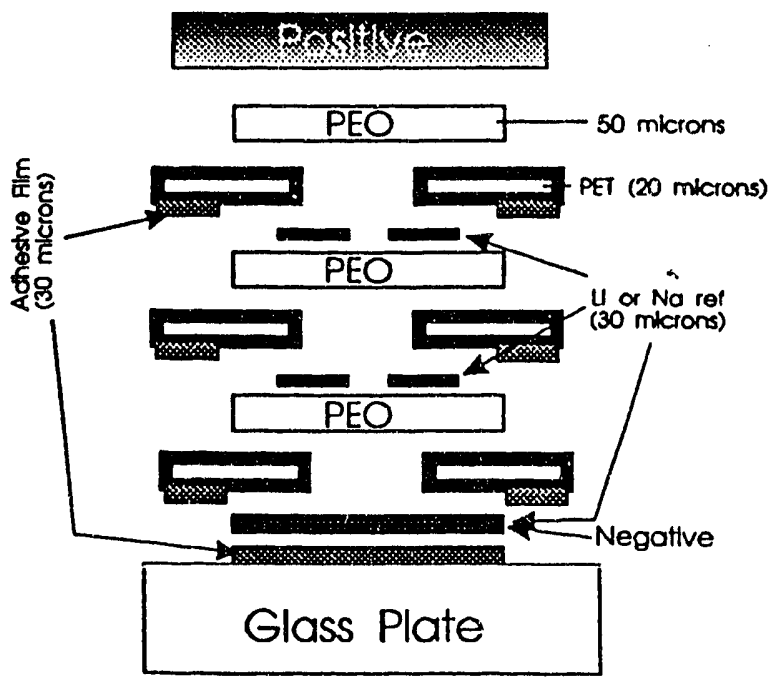

Figure 2. Assembly of solid polymer 4-probe cell. 


\section{ANALYSIS}

\section{Effect of Double Layer Charging}

Under the assumption that mass transfer is infinitely fast, the potential drop across an interface at time $t$ after a constant current $I$ is applied to the interface, can be expressed as

$$
\eta(t)=\eta_{a}\left[1-\exp \left(-\frac{t}{C_{d l} R_{c t}}\right)\right]
$$

Eq. 1

where $\eta_{\alpha}$ is the charge transfer overpotential, $C_{d}$ is the double layer capacitance of the interface, and $R_{c}$ is the charge transfer resistance of the interface. When the current due to double layer charging vanishes $\left(i_{d l} \rightarrow 0\right)$, the applied current approaches the current due to faradaic processes $\left(I_{f}\right)$ and the interfacial overpotential, $\eta(t)$, approaches the steady-state charge transfer overpotential, $\eta_{\star}$, which is related to the applied current, $I$, as

$$
\frac{I}{A}=\frac{I_{f}}{A}=i_{0}\left\{\exp \left[\frac{\alpha_{a} F \eta_{\alpha}}{R T}\right]-\exp \left[\frac{\alpha_{c} F \eta_{\alpha}}{R T}\right]\right\}
$$

where $A$ is the surface area of the electrode, $i_{o}$ is the exchange current density, $R$ is the gas constant, $T$ is the absolute temperature, $F$ is Faraday's constant, and $\alpha_{a}$ and $\alpha_{c}$ are the anodic and cathodic transfer coefficient, respectively. This equation also implies that $\eta_{c t}$ is defined under the conditions that there is no mass transfer limitation, i.e., the concentrations of the electroactive species at the surface is identical or very close to the concentrations in the bulk.

The charge transfer resistance can be expressed as

$$
R_{c t}=\left(\frac{\partial \eta}{\partial t}\right)_{i \rightarrow 0}=\frac{R T}{i_{0}\left(\alpha_{a}+\alpha_{c}\right) F}
$$

Eq. 3

\section{Effect of Mass Transfer}

Although the effects of mass transfer can be minimized by suitable choice of experimental conditions, its contribution cannot always be igtiored. As soon as current is passed through an interface, a gradient in concentration of electroactive species is generated at the interface, leading to concentration polarization. Equation 1 can be modified to include the effects of mass transfer as

$$
\eta(t)=\eta_{c}\left[1-\exp \left(-\frac{t}{C_{d l} R_{c t}}\right)\right]+\eta_{m t}(t)
$$

where $\eta_{m t}(t)$ is the overpotential due to mass transfer. 
As a first approximation, the mass transfer overpotential due to the concentration gradient of species $i$ can be approximated by

$$
\eta_{m t, i}(t)=\left(\frac{R T}{\alpha_{i} F}\right) \ln \left(\frac{c_{i}(0, t)}{c_{i}^{*}}\right)
$$

where $\alpha_{i}$ is the anodic or cathodic transference coefficient for the electrochemical reactions involving species $i, c_{i}{ }^{*}$ is the concentration of species $i$ in the bulk phase, and $c_{i}(0, t)$ is the concentration of species $i$ at the interface at time $t$.

When a constant current, $I$, is applied to an interface, the concentration of species $i$ at any given time can be expressed $\mathrm{as}^{4}$

$$
c_{i}(0, t)=c_{i}^{*} \pm \frac{2 I t_{i}(t)^{\frac{1}{2}}}{n_{i} F A\left(\pi D_{1}\right)^{\frac{1}{2}}}
$$

where $D_{i}$ is the diffusion coefficient of species $i, t_{i}$ is the transference number of species $i$, and $n_{i}$ is the number of electrons transferred in the reaction involve species $i$. The use of the \pm sign depends on the nature of the electrochemical reaction; a + sign is appropriate for an electrochemical reaction which generates species $i$, while a - sign is necessary for a reaction which consumes species $i$. This follows since the polarity of the overpotential is such to increase the overpotential at the interface, thereby diminishing that electrochemical process.

Accordingly, the overpotential due to mass transfer of species $i$ can be expressed in terms of the applied current as

$$
\eta_{m, t i}=\left(\frac{R T}{\alpha_{i} F}\right) \ln \left[1 \pm \frac{2 I t_{i} t^{\frac{1}{2}}}{n_{i} F A c_{i}^{*}\left(\pi D_{i}\right)^{\frac{1}{2}}}\right]
$$

If the mass transport of more than one species contribute to the concentration overpotential, the total overpotential due to mass transfer will be the summation over all the species:

$$
\eta_{m t}(t)=\sum_{i} \eta_{i m t, i}(t)
$$

Eq. 8

\section{Potential Response to Sequential Current Pulses}

A simplified 4-probe cell and corresponding equivalent circuit is shown in figure 3. 

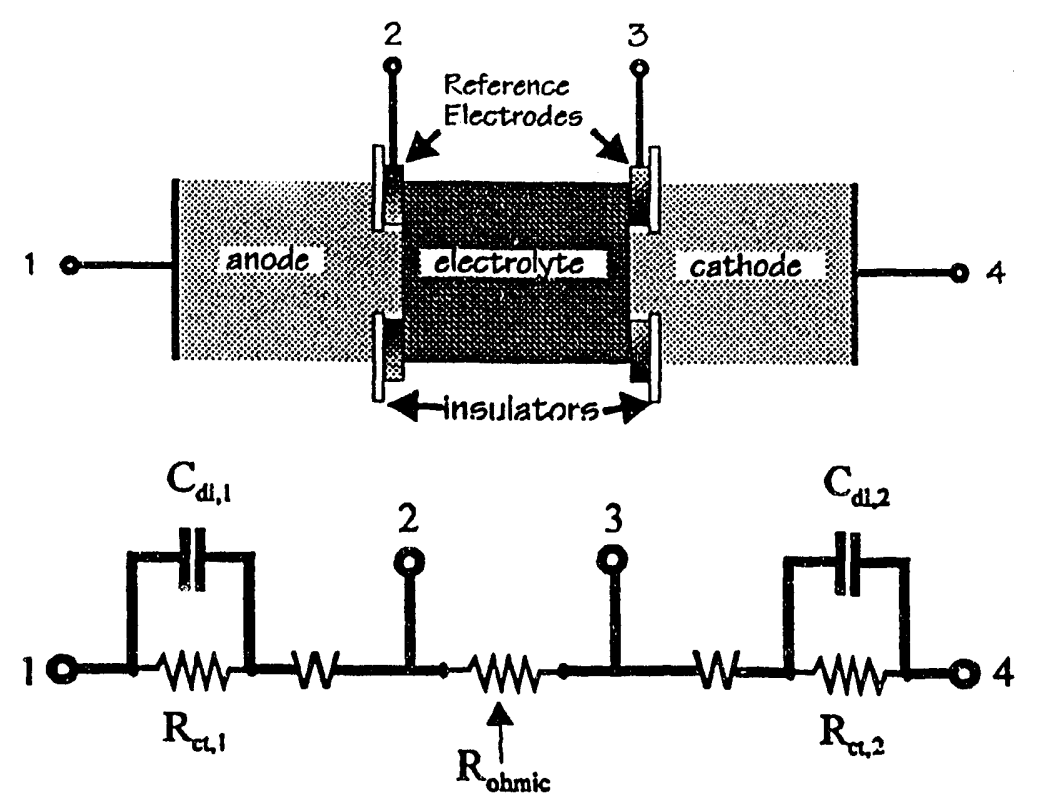

Figure 3. Simplified 4-probe cell and equivalent circuit.

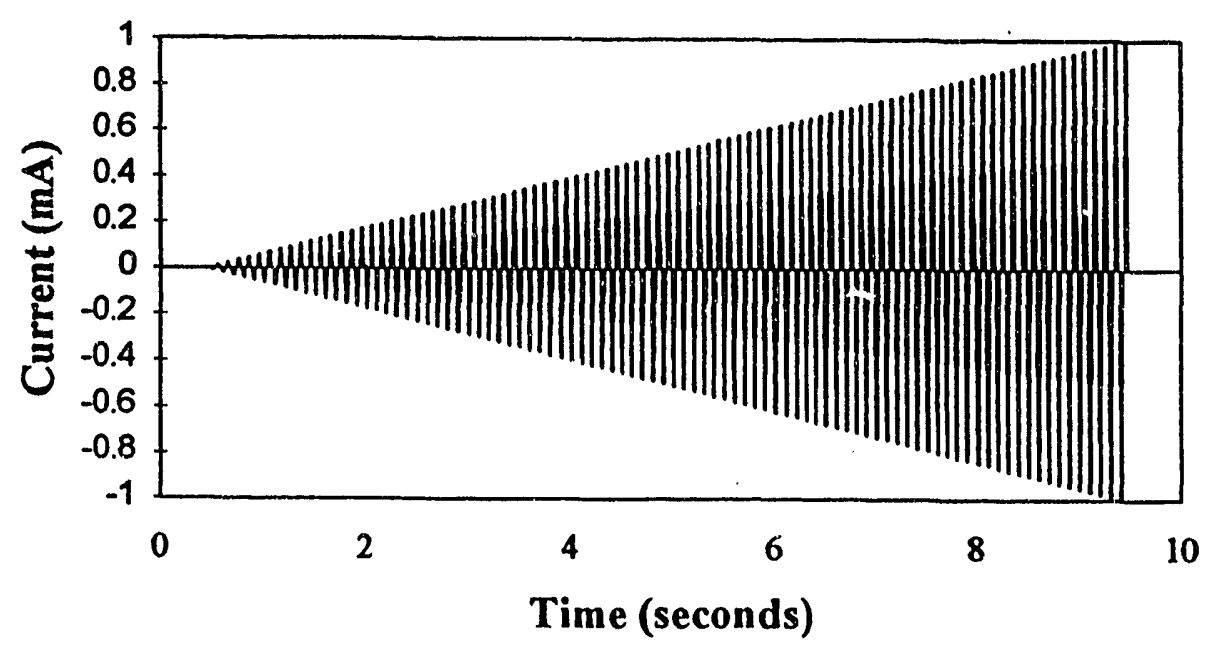

Figure 4. Bipolar current array applied to 4-probe cell; 180 square wave current pulses.

When symmetric, sequential current pulses as shown in Figure 4 are applied to the cell the corresponding potential response for each pulse $\left(0<1 \leq \tau_{0}\right)$ can be described as

$$
\begin{gathered}
V_{1-2}(t)=\eta_{c, 1}\left[1-\exp \left(-\frac{t}{\dot{\tau}_{1}}\right)\right] \pm \sum_{i} \frac{R T}{\alpha_{i} F} \ln \left[1 \pm\left(\frac{t}{\dot{\tau}_{i, 1}}\right)^{\frac{1}{2}}\right] \\
V_{2-3}=I R_{\text {ohmic }} \\
V_{3-4}(t)=\eta_{a, 2}\left[1-\exp \left(-\frac{t}{\dot{\tau}_{2}}\right)\right] \pm \sum_{i} \frac{R T}{\alpha_{i} F} \ln \left[1 \pm\left(\frac{t}{\dot{\tau}_{i, 2}}\right)^{\frac{1}{2}}\right]
\end{gathered}
$$

Eq. 10

Eq. 11 
where the relaxation time associated with double layer charging at each interface can be expressed as

$$
\begin{aligned}
& \dot{\tau}_{1}=C_{d l, 1} R_{c t, 1}=\left[\left(\frac{1}{\alpha_{a}+\alpha_{c}}\right)\left(\frac{R T}{F}\right) \frac{C_{d l}}{i_{o}}\right]_{\text {interface 1 }} \\
& \dot{\tau}_{2}=C_{d l, 2} R_{c t, 2}=\left[\left(\frac{1}{\alpha_{a}+\alpha_{c}}\right)\left(\frac{R T}{F}\right) \frac{C_{d l}}{i_{o}}\right]_{\text {interjace 2 }}
\end{aligned}
$$

and the transition time associated with mass transport of species $i$ near each electrode surface can be defined as

$$
\begin{aligned}
& \left(\dot{\tau}_{i, 1}\right)^{\frac{1}{2}}=\left[\frac{n_{i} F A c_{i}^{*}\left(\pi D_{i}\right)^{\frac{1}{2}}}{2 I t_{i}}\right]_{\text {interfacel }} \\
& \left(\dot{\tau}_{i, 2}\right)^{\frac{1}{2}}=\left[\frac{n_{i} F A c_{i}^{*}\left(\pi D_{i}\right)^{\frac{1}{2}}}{2 I t_{i}}\right]_{\text {inderface 2 }}
\end{aligned}
$$

As can be readily seen, the two time constants are quite different; $\tau^{\prime}$ is independent of the applied current and depends on the double layer capacitance and the exchange current density. On the other hand, $\tau^{\prime \prime}$ depends on the applied current density, the diffusion coefficient, transference number, and the concentration of the electroactive species.

\section{Selection of Appropriate Pulse Width}

Since the electrochemical response of an interface is strongly time-dependent, it is important to select appropriate pulse width in order to minimize or eliminate the complications due to mass transport and double layer charging. Accordingly, the duration of the current pulse should be long enough that the current due to double layer charging is reasonably small, and yet short enough that the concentration of the electroactive species at the electrode surface is reasonably close to the concentration in the bulk.

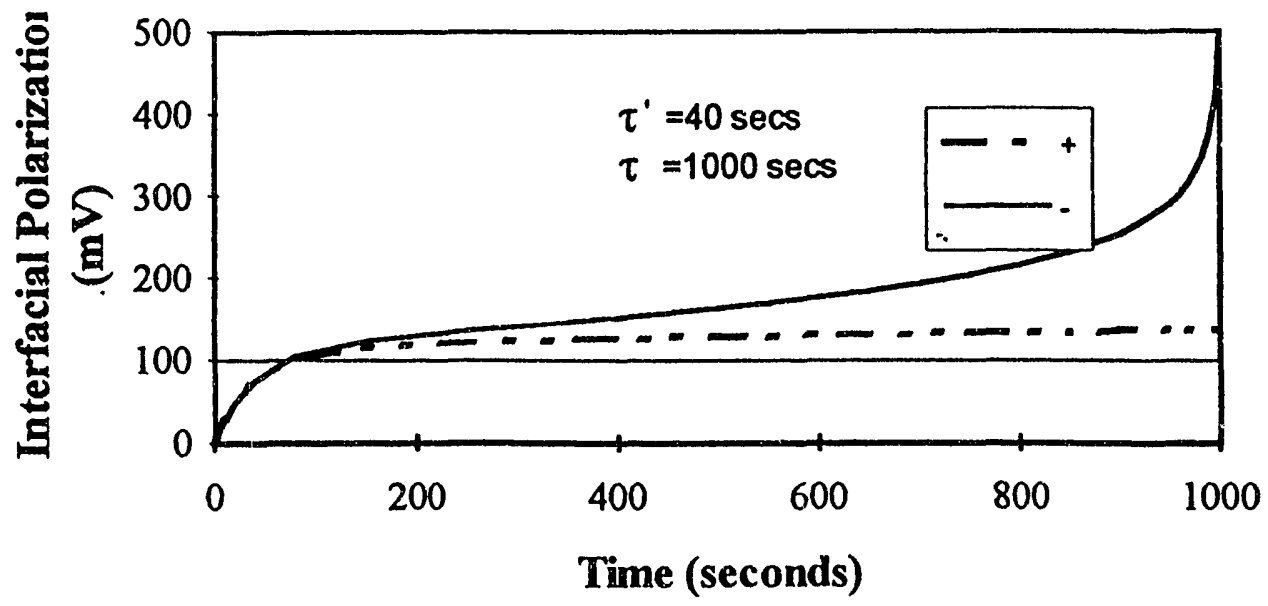

Figure 5. Combined effects of double layer charging and mass transfer on the interfacial potential over the entire time domain 


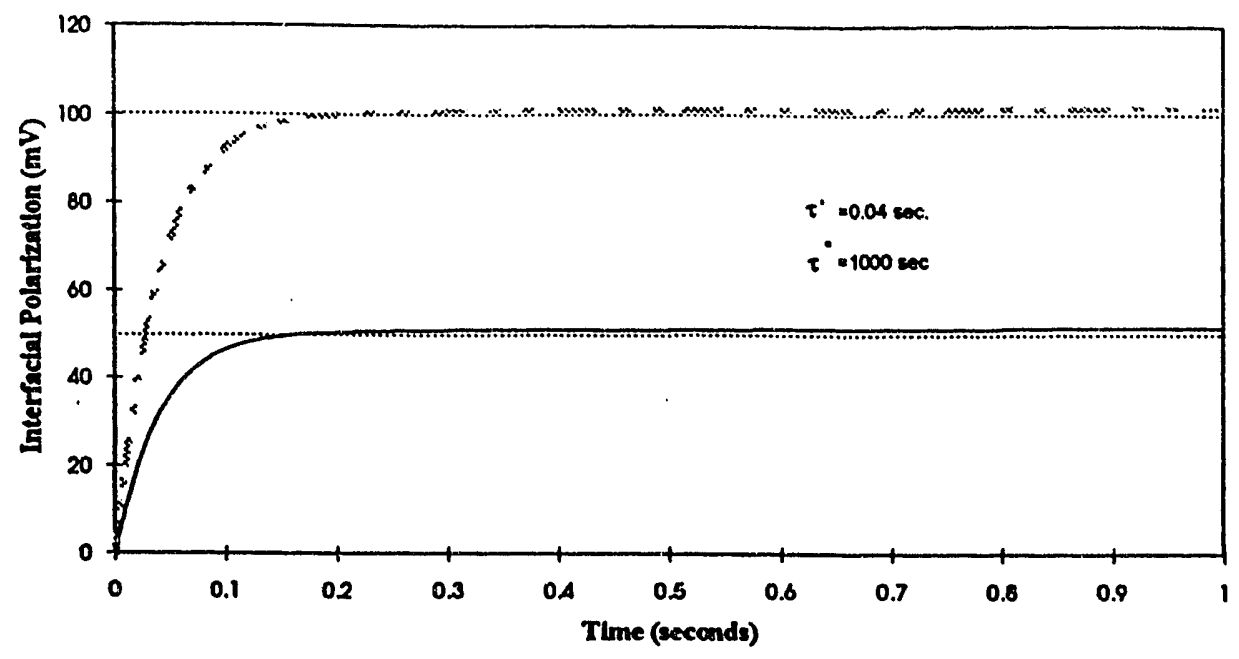

Figure 6. Effects of double layer charging and mass transfer on the interfacial potential at a realistic value for the double layer time constant and experimental time window.

Shown in figures 5 and 6 are the combined effects of double layer charging and mass transfer on interfacial potentials. Figure 5 shows the effect over the entire time domain. In order to the effect of double layer charging, the time constant was greatly exaggerated to be 40 seconds, much longer than experimentally observed values. Further, the effect of mass transfer was exaggerated by assuming the transition time constant for mass transfer to be 1000 seconds, much shorter than experimentally observed values. For this case, it would be exceedingly difficult to obtain accurate values of the interfacial potential at any pulse width. A more realistic case is shown in figure 6 where the time constant for double layer charging is taken to be $40 \mathrm{msec}$, and the interfacial potential is shown over the time window where 40 $m s e c \ll t \ll 1000 \mathrm{sec}$. In this realistic situation, it is readily apparent that a very accurate measure of the interfacial potential is obtained at times greater than $200 \mathrm{msec}$ and less than about $2000 \mathrm{msec}$. The double layer relaxation time constant can be readily determined by impedance spectroscopy, or by measuring the potential response in the DC pulse experiment as a function of time. The relaxation time constant for mass transfer is easily determined by chronopotentiometry.

The time constant for double layer charging is typically orders of magnitude smaller than that for mass transfer, particularly when the applied current density is relatively small. In order to minimize the error introduced by double layer charging, the pulse width should be kept a few times greater than the relaxation time of the double layer and much smaller than the transition time for mass transfer.

\section{RESULTS}

\section{Four Probe DC Techniques for Solid Polymer Cells}

A schematic view of the 4-probe is shown in figure 1 . When a constant current, or pulse sequence (fig. 4), is applied across the cell, the ohmic potential drop across the center electrolyte film can be determined as

$$
V_{\text {ohmic }}=V_{2-3}
$$


The anodic overpotential can be determined as

$$
\eta_{a}=V_{1-2}-V_{\text {ohmic }}
$$

while the cathodic overpotential is determined by

$$
\eta_{c}=V_{1-4}-V_{1-3}-V_{\text {ohmic }}
$$

Eq. 18

In this way, the potential drop across the interfaces and across the electrolyte can be cleanly separated.

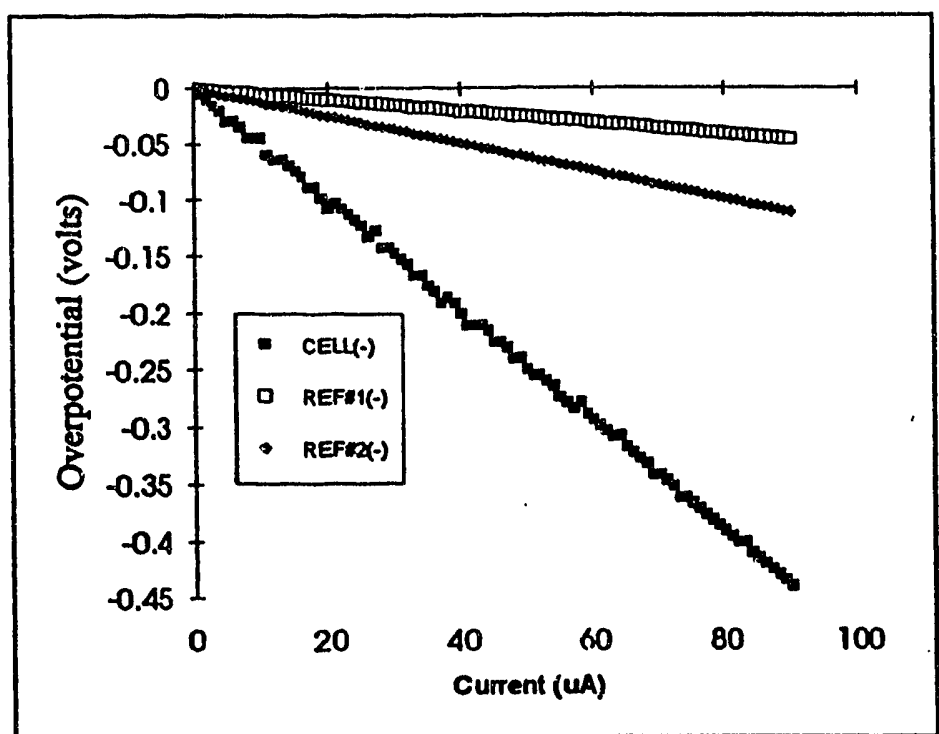

Figure 7. Experimentally observed values of the potential response of a $\mathrm{Li} /(\mathrm{aPEO}) \times 3 / \mathrm{X} 1$ four probe cell to a bipolar pulse sequence of the type shown in figure 4 (cathodic response shown).

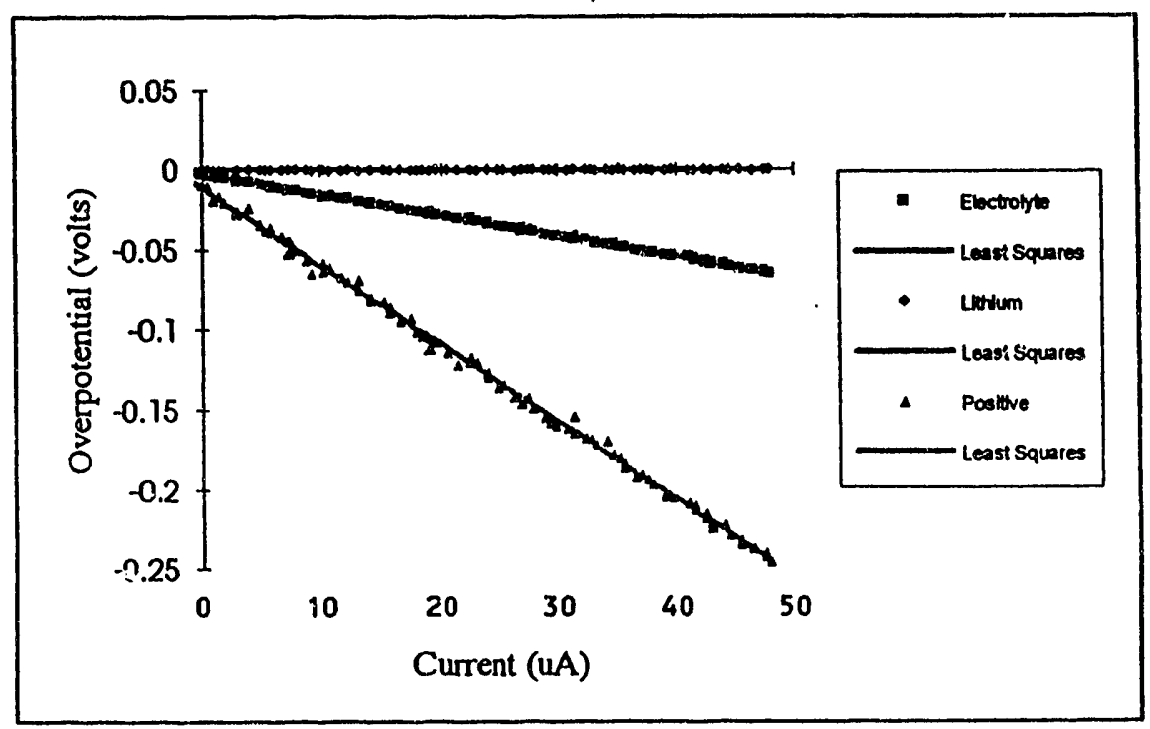

Figure 8. Interfacial polarization of anode and cathode and ohmic drop across electrolyte as a function of current applied across $\mathrm{Li} /(\mathrm{aPEO}) \times 3 / \mathrm{X} 14$-probe cell (from data in figure 7).

The potential response of a $\mathrm{Li} / \mathrm{aPEO} / \mathrm{X} 1$ iell of the type shown in figure 1 to a bipolar puise sequence (ñg. 4) is shown in figure 7; only the negative values of the bipolar response of the cell are shown. The polarization of the electrolyte, lithium electrode-electrolyte interface, and positive electrode can be determined according to equations 16-18; and is shown in figure 
8. The corresponding resistances can be calculated from the slopes of the polarization curves in figure 8. In a similar fashion, activation parameters for the various components of the cell can be determined by making the same measurements over a range of temperatures. In figure 9 , polarization measurements were made on a 4-probe $\mathrm{Li} / \mathrm{aPEO} / \mathrm{Li}_{2} \mathrm{Xl}$ cell from ambient to 70 ${ }^{\circ} \mathrm{C}$, and the resistances of the lithium-aPEO interface, aPEO electrolyte, and composite positive electrode were determined over that temperature range as described above. In this case, the close similarity in the temperature dependence electrolyte and positive electrode resistance indicates that the performance of the positive electrode is largely due to the relatively low conductivity of the electrolyte. In fact, the magnitude of the electrolyte resistance is such that the positive electrode polarization is linear with current (ohmic), rather than exhibiting a Tafel-like polarization.

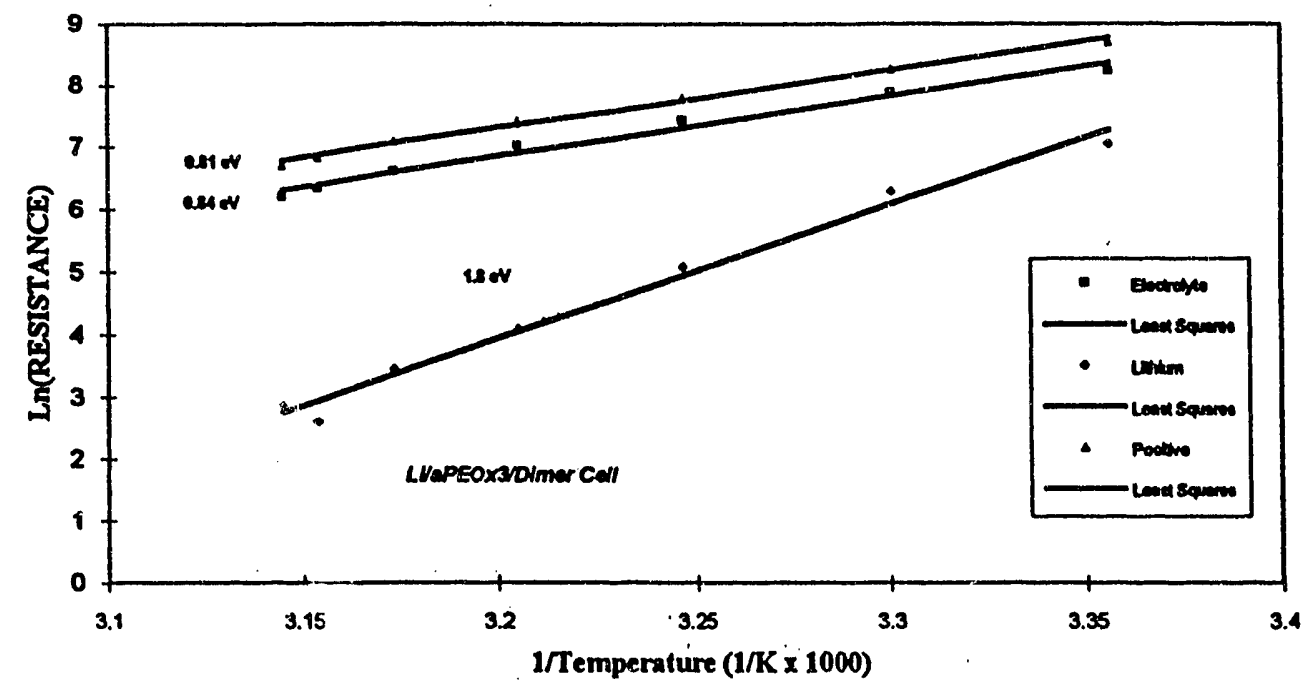

Figure 9. Arrhenius plot of the resistances of the electrolyte and electrode interfaces in a $\mathrm{Li} /$ aPEO $3 / \mathrm{Li}_{2} \mathrm{XI}$ cell, as determined from 4-probe pulse polarization measurements.

Figure 10 shows the room temperature polarization response of a positive electrode in a Li/gelled PEO)XI cell as determined from 4-probe pulse measurements. In this case Tafel behavior of the positive electrode is cleanly separated from the overall cell response. The exchange current and ohmic drop.in the positive electrode can then be calculated by a nonlinear curve fit of the data as shown in figure 10.

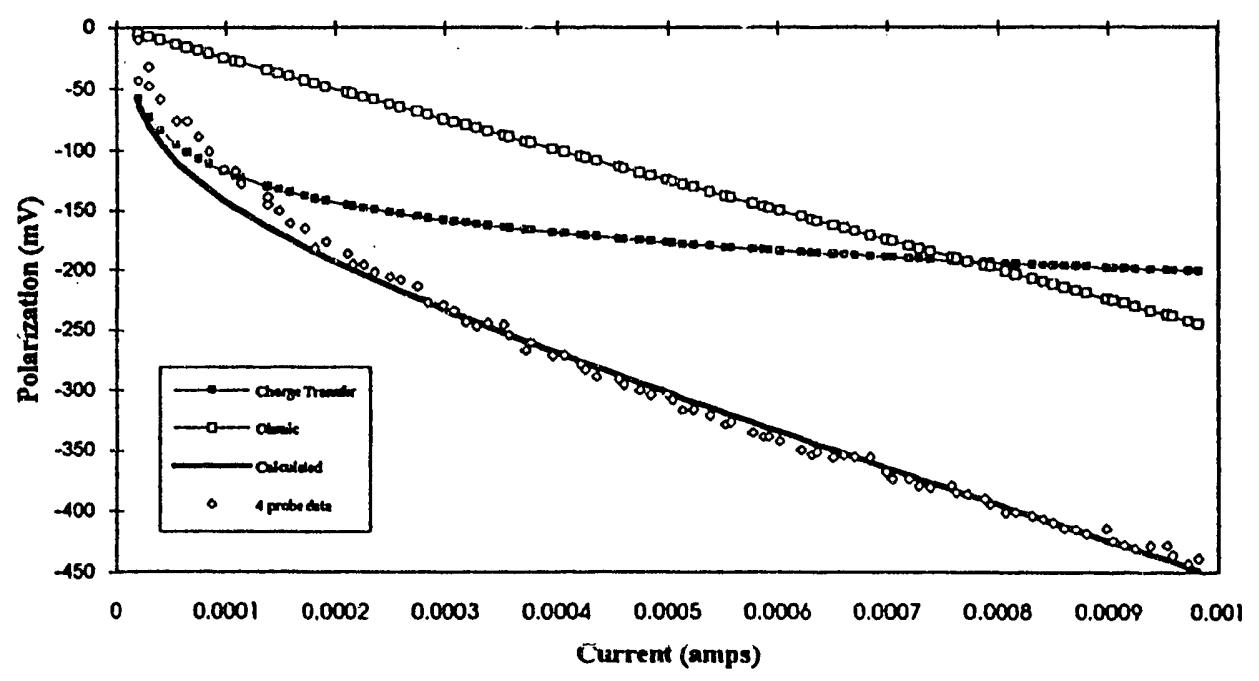

Figure 10. Positive electrode polarization in a $\mathrm{Li} / \mathrm{PEO}$ (plasticized)/X1 cell as determined from 4 probe measurements; calculated fit corresponds to $\alpha=0.35, R_{o h m i c}=250 \Omega$, and $i_{o}=4 \times 10^{-6}$. 
The utility of 4-probe cells for steady-state measurements is shown in figure 11. The various contributions to the overall polarization of a $\mathrm{Na} / \mathrm{PEO} / \mathrm{X} 5$ cell at constant current as a function of time is shown in figure 11. The 4-probe technique allows clear identification of the controlling factors to cell performance. In this cell there is evidence of reaction at the $\mathrm{Na} / \mathrm{PEO}$ interface, as well as strong polarization of the positive electrode indicating mass transfer limitations. To improve cell performance the positive electrode must be improved, and the stability of the anode/electrolyte interface must be increased.

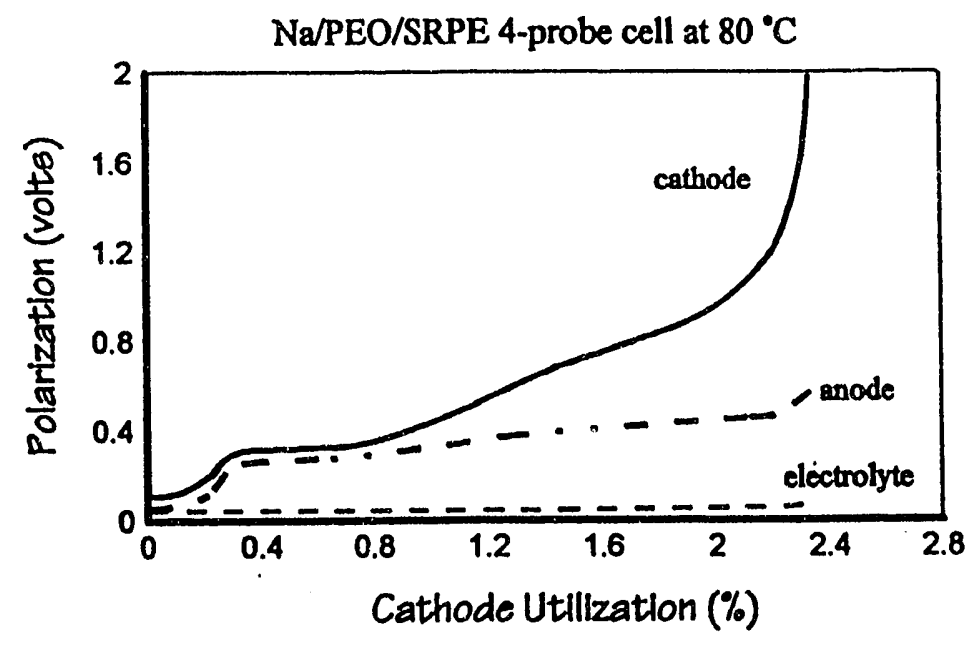

Figure 11. Overpotentials observed directly in a $\mathrm{Na} /(\mathrm{PEO}) \times 3 / \mathrm{X} 5$ four-probe cell under constant-current control.

\section{CONCLUSIONS}

The use of 4-probe techniques provide powerful tools to determine the limiting factors to performance in solid-state electrochemical devices. These methods are particularly useful for quick diagnosis of the rate limiting components of an electrochemical cell. In addition, careful design of the experimental cell and appropriate selection of current pulse width allows evaluation of fundamental kinetic and transport properties, as well as activation parameters.

\section{ACKNOWLEDGMENT}

This research was supported by the Assistant Secretary for Conservation and Renewable Energy, Deputy Assistant Secretary for Utility Technologies, Office of Energy Management, Advanced Utility Concepts Division of the U.S. Department of Energy under Contract No. DE-AC03-76SF00098.

\section{REFERENCES}

${ }^{1}$ M. Liu, S.J. Visco, and L.C. De Jonghe, J. Electrochem. Soc., 138, 1891 - 1895 (1991).

${ }^{2}$ M. Liu, S.J. Visco, and L.C. De Jonghe, J. Electrochem. Soc., 138, 1896 - 1991 (1991).

${ }^{3}$ S.J. Visco, M. Liu, and L.C. De Jonghe, Mol. Cryst. Liq. Cryst., 190, 185 (1990).

${ }^{4}$ A.J. Bard and L.R. Faulkner, "Electrochemical Methods, Fundamentals and Applications", Wiley, New York, 1980. 

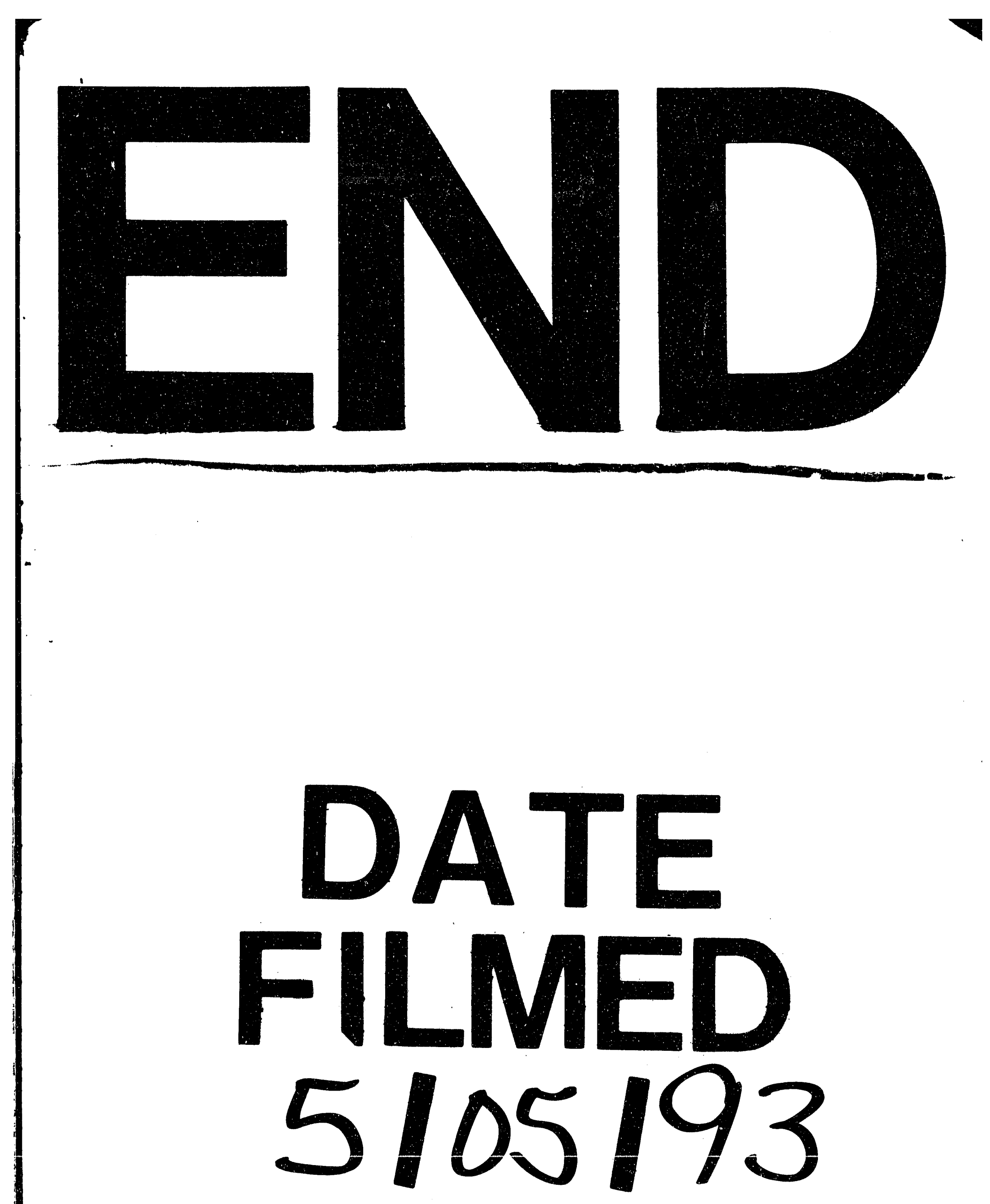